Rev. Bras. Saúde Prod. Anim., Salvador, v.16, n.4, p.784-795 out../dez.., 2015 http://www.rbspa.ufba.br

\title{
Diversidade fenotípica de linhagens de ovinos Santa Inês por meio de análise multivariada
}

\author{
Multivariate analysis of phenotypic diversity in Santa Ines sheep
}

\author{
TEIXEIRA NETO, Milton Rezende ${ }^{1 *}$; CRUZ, Jurandir Ferreira da ${ }^{2}$; CARNEIRO, \\ Paulo Luiz Souza ${ }^{3}$; MALHADO, Carlos Henrique Mendes ${ }^{3}$; BARBOSA, Joselito \\ Araújo $^{4}$; SOUZA, Luiz Eduardo Barreto de ${ }^{1}$
}

\footnotetext{
${ }^{1}$ Universidade Estadual do Sudoeste da Bahia, Pós-Graduação em Zootecnia, Itapetinga, Bahia, Brasil.

${ }^{2}$ Universidade Estadual do Sudoeste da Bahia, Departamento de Fitotecnia e Zootecnia, Vitória da Conquista, Bahia, Brasil.

${ }^{3}$ Universidade Estadual do Sudoeste da Bahia, Departamento de Ciências Biológicas, Jequié, Bahia, Brasil.

${ }^{4}$ Associação dos Criadores de Caprinos e Ovinos da Bahia, Bahia, Brasil.

*Endereço para correspondência: rezendeteixeira@yahoo.com.br
}

\section{RESUMO}

Objetivou-se com este estudo avaliar a diversidade fenotípica de linhagens de ovinos Santa Inês. Doze medidas corporais foram realizadas em 502 fêmeas adultas descendentes de 34 reprodutores pertencentes a 15 linhagens da raça Santa Inês, registrados junto à associação de criadores: Altura Anterior, Altura Posterior, Altura das Pernas, Altura do Tórax, Circunferência Torácica, Circunferência da Canela, Comprimento do Corpo, Comprimento do Pescoço, Comprimento de Orelha, Comprimento da Face, Comprimento do Chanfro e Largura da Face. Os dados foram submetidos à análise de Variáveis Canônicas e à análise de agrupamento pelo método da ligação média entre grupos. As três primeiras Variáveis Canônicas explicaram $81,01 \%$ da variação total. As linhagens 1,10 e 11 foram as mais homogêneas, enquanto que as linhagens 3, 6, 9, 12 e 15 apresentaram grande variabilidade intrapopulacional. As linhagens 11 e 15 foram as que apresentaram maior distância (Mahalanobis). A análise de agrupamento mostrou a formação de três grupos. As cinco medidas corporais com maior importância relativa (Circunferência Torácica, Circunferência da Canela, Comprimento da Face, Comprimento de Pescoço e Comprimento de Chanfro), consideradas conjuntamente, explicaram $76,89 \%$ da diversidade fenotípica entre as linhagens. As linhagens de ovinos Santa Inês apresentam alta diversidade fenotípica, sendo que as características relacionadas à produção e à caracterização racial apresentam importância semelhante na diferenciação entre linhagens.

Palavras-chave: análise multivariada, biometria corporal, distância de Mahalanobis, ovinos deslanados

\section{SUMMARY}

This study aimed at evaluating the phenotypic diversity of Saint Ines sheep strains. Twelve body measurements were carried out in 502 adult females, descendants from 34 reproducers belonging 15 strains of Santa Ines breed, registered at association of creators: anterior height, posterior height, legs height, chest height, thoracic circumference, shin circumference, body length, neck length, ear length, face length, chamer length and face width. The data were submitted to the canonic variable analysis and cluster analysis by means of the method Unweighted Pair-Group Method Using Arithmetic Averages. The first three canonical variables explained $81.01 \%$ of the total variation. Strains 1,10 and 11 were the most homogeneous, whereas strains 3, 6, 9, 12 and 15 presented large intra-population variability. Strains 11 and 15 presented the longer distance (Mahalanobis). The cluster analysis showed the formation of three groups. The five body measurements with higher relative importance (thoracic circumference, 
shin circumference, face length, neck length, and chamfer length), jointly considered, accounted for $76.89 \%$ of the phenotypic diversity between the lineages. The strains of Santa Ines sheep present high phenotypic diversity, and the characteristics related to production and racial characterization are similarly important in the differentiation between the strains.

Keywords: body measurements, Mahalanobis distance, multivariate analysis, wooless sheep

\section{INTRODUÇÃO}

A raça Santa Inês, formada no Nordeste brasileiro, mais especificamente no estado da Bahia (PAIVA et al., 2005), é resultante, provavelmente, de acasalamentos entre ecótipos africanos e as raças Morada Nova, Rabo Largo, Somalis e Bergamácia.

$\mathrm{Na}$ fase inicial de formação da raça, foram priorizadas as características raciais em detrimento daquelas voltadas para produção, no entanto, existem atualmente alguns programas de melhoramento genético específicos para a raça Santa Inês visando à avaliação e melhoramento das características produtivas (CARNEIRO et al., 2006).

A diversidade fenotípica pode ser um indicador de diferenças adaptativas ou seletivas entre populações (BIAGIOTTI et al., 2013), sendo que a diversidade das características quantitativas entre populações permite fazer inferências sobre o grau de adaptação a determinado ambiente ou sobre a especialização produtiva (DARAMOLA \& ADELOYE, 2009).

A manutenção da diversidade intrarracial é fundamental para sobrevivência das populações naturalizadas e, no caso dos programas de melhoramento genético, a garantia de que se possa estabelecer pressão de seleção e aumento de produção em função do sistema de criação (PIRES et al, 2013; DOSSA et al., 2007). A diversidade fenotípica de ovinos, estimada por meio dos marcadores biométricos, pode fornecer informações importantes a respeito do grau de divergência entre grupos estudados (YAKUBU \& IBRAHIM, 2011; BIAGIOTTI et al., 2014).

Considerando que as características de importância econômica estão correlacionadas, com magnitudes e sentidos variáveis, a utilização de técnicas de análise multivariada é a mais recomendada no estudo da diversidade, pois quando existe interdependência, as análises univariadas não apresentam a versatilidade de aproveitar as correlações existentes (TORRES FILHO et al., 2005). Desta forma, buscando gerar informações estratégicas para programas de melhoramento genético, este estudo teve como objetivo avaliar a diversidade fenotípica de ovinos da raça Santa Inês.

\section{MATERIAL E MÉTODOS}

Nesse estudo foram mensuradas 502 fêmeas ovinas da raça Santa Inês registradas, adultas, com idade superior a dois anos, filhas de 34 reprodutores pertencentes a 15 linhagens, oriundas de seis fazendas no estado da Bahia, localizadas em cinco municípios: duas em Ipirá (183 animais), duas em Senhor do Bonfim (112 animais), uma Ribeirão do Largo (97 animais) e uma em Serra Preta (110 animais). Consideraram-se como linhagens os grupos de indivíduos descendentes dos principais reprodutores da raça, de acordo com a Associação de Criadores de Caprinos e Ovinos da Bahia (ACCOBA) (Tabela 1). 
Rev. Bras. Saúde Prod. Anim., Salvador, v.16, n.4, p.784-795 out../dez.., 2015 http://www.rbspa.ufba.br ISSN 15199940

Tabela 1. Número de animais por linhagem de ovinos da raça Santa Inês

\begin{tabular}{cccc}
\hline Linhagem & Número de animais & Linhagem & Número de animais \\
\hline 1 & 31 & 9 & 28 \\
2 & 41 & 10 & 28 \\
3 & 31 & 11 & 27 \\
4 & 43 & 12 & 33 \\
5 & 51 & 13 & 30 \\
6 & 48 & 14 & 31 \\
7 & 27 & 15 & 24 \\
8 & 29 & - & - \\
\hline
\end{tabular}

As medidas corporais de caracterização racial realizadas foram: Altura das Pernas (PR), Comprimento do Pescoço (CP), Orelha (CO), Face (CF) e do Chanfro $(\mathrm{CH})$ e Largura da Face (LF). Enquanto que as medidas corporais relacionadas à produção foram: Altura Anterior (AA), Posterior (AP), e do Tórax (AT), Circunferência Torácica (CT) e da Canela (CN), Comprimento do Corpo (CC).

A aferição do Comprimento do Corpo foi feita medindo-se a distância entre a articulação cérvico-torácica e a base da cauda na primeira articulação intercoccígea, com o animal mantido em posição correta de aprumos. A Altura Anterior foi medida entre o ponto mais alto da região interescapular e o solo, a Altura Posterior, entre a tuberosidade sacral do ílio e o solo, e a Altura Torácica, entre o piso do tórax até a cernelha. A Circunferência Torácica foi feita contornando o tórax, tomando-se como base a cernelha e as axilas. O Comprimento do Pescoço foi obtido medindo-se a distância entre a porção cranial do arco dorsal do atlas e o terço médio da borda cranial da escápula. A Circunferência da Canela foi feita contornando o terço médio da região metacarpiana do membro esquerdo. O Comprimento de Orelha foi feito medindo a distância da base orelha até a sua extremidade. O Comprimento da Face foi obtido pela distância entre a extremidade proximal da cabeça que coincide com a crista da nuca e a parte medial ou central da arcada incisiva inferior, e a Largura da Face pela distância entre a parte livre da borda supra-orbital até a borda supra-orbital esquerda. O Comprimento do Chanfro foi feito medindo da ponta do nariz à linha que une os rebordos internos dos olhos. A Altura das Pernas foi calculada pela diferença entre as medidas AA e AT.

$\mathrm{A}$ aferição das medidas $\mathrm{CC}, \mathrm{AA}, \mathrm{AP}$, AT e CF foram realizadas com o auxílio da Bengala de Lydtin, enquanto que a aferição da $\mathrm{CT}, \mathrm{CN}, \mathrm{CP}$ e $\mathrm{CH}$ foram feitas com o auxílio de uma fita métrica. Para obtenção da LF, foi utilizado um paquímetro e para $\mathrm{CO}$ uma régua milimetrada, conforme sugerido por Searle et al. (1989).

Os dados biométricos das diferentes linhagens foram submetidos à análise de variância em função da linhagem, e comparação das médias pelo teste Scottknott $(\mathrm{p}<0,05)$. As correlações entre as medidas corporais foram avaliadas por meio da correlação linear de Pearson $(p<0,05)$. Ambas as análise realizadas mediante o programa Genes versão 6.0 (CRUZ, 2008).

Para determinar a diversidade fenotípica entre linhagens, foi utilizada a análise discriminante, mediante o procedimento 
Candisc do programa estatístico SAS, versão 8.0 (SAS, 2003) e o agrupamento pelo método hierárquico Unweighted Pair Group Method With Arithmetic Mean (UPGMA), mediante o programa Genes (versão 6.0).

A identificação e descarte das características redundantes (aquelas que explicaram a mesma variação entre linhagens) foram feitas por meio de análise discriminante, sendo descartadas todas as medidas com maior autovetor nas Variáveis Canônicas de autovalor $<0,70$, conforme sugerido por Jolliffe (1972). Ainda como critério de descarte, foi considerado os coeficientes de correlação de Pearson entre as medidas. Após o descarte das medidas redundantes, procedeu-se nova análise discriminante com as medidas selecionadas.

Para o agrupamento das linhagens pelo método UPGMA, adotou-se a distância generalizada de Mahalanobis $\left(\mathrm{D}^{2}\right)$ como medida de dissimilaridade. $\mathrm{O}$ ponto de corte do dendrograma foi definido pela média da matriz da $\mathrm{D}^{2}$ e para avaliar a consistência do agrupamento calculouse o coeficiente de correlação cofenética.

A importância relativa das características para a divergência foi obtida por meio da partição dos componentes $\mathrm{D}^{2}$, relativos a cada característica, no total da dissimilaridade observada, conforme sugerido por Singh (1981).

\section{RESULTADOS E DISCUSSÃO}

Os Coeficientes de Variação (CV) para as características analisadas oscilaram entre $3,25 \%$ e $6,85 \%$, sendo que as medidas que obtiveram maiores $\mathrm{CV}$ foram o Comprimento de Pescoço, Altura das Pernas e Comprimento de
Orelha (Tabela 2). Os maiores CV verificados para estas três medidas refletem, provavelmente, a variabilidade dos genótipos que deram origem à raça Santa Inês. Os ovinos Santa Inês são resultantes do acasalamento entre ecótipos africanos e as raças Morada Nova, Rabo Largo e Somalis, todas caracterizadas por possuírem orelhas pequenas, pescoço curto e porte pequeno ou mediano. Por outro lado, houve contribuição significativa da raça Bergamácia, a qual apresenta orelhas longas, pescoço comprido e grande porte.

A linhagem 15 foi a mais alta e a mais comprida, sugerindo influência da raça Bergamácia, enquanto que a linhagem 11 foi a de menor estatura e corpo mais curto denotando influência da raça Somalis. A linhagem 3 apresentou porte mediano, porém com orelhas pequenas, o que pode ser indicativo de influência das raças Rabo Largo e Morada Nova (Tabela 2). As demais linhagens se alternaram nas diferentes medidas, evidenciando grande diversidade fenotípica dentro da raça Santa Inês.

Em estudo da caracterização morfométrica dos ovinos Santa Inês criados no estado do Piauí, Biagiotti et al. (2013), verificou-se que as populações das oito microrregiões analisadas apresentaram características fenotípicas diferenciadas entre si e, semelhantemente ao presente estudo, encontraram elevada diversidade dentro da raça Santa Inês.

As medidas que expressam o padrão racial $(\mathrm{CP}, \mathrm{CH}, \mathrm{CO}, \mathrm{LF}, \mathrm{CF}$ e $\mathrm{PR})$ apresentaram correlações baixas $(0,00$ $<r<0,30$ ) ou não significativas ( $p>0,05)$. Por outro lado, as demais medidas (AA, AT, AP, CC, CT e CN) apresentaram correlações moderadas ou elevadas $(0,50 \leq \mathrm{r}<0,91)$ (Tabela 3$)$.

Em estudo com bovinos de corte, foram encontradas correlações moderadas e 
Rev. Bras. Saúde Prod. Anim., Salvador, v.16, n.4, p.784-795 out../dez.., 2015 http://www.rbspa.ufba.br ISSN 15199940

elevadas entre $\mathrm{CN}$ e peso da carcaça quente $(0,42)$, peso vivo $(0,60)$, CT $(0,72)$ e CC $(0,63)$ (GILBERT et al., 1993). De forma semelhante, em estudo com a espécie caprina, foram encontradas correlações elevadas entre $\mathrm{CN}$ e peso vivo $(0,80), \mathrm{CT}(0,69)$ e $\mathrm{CC}$ $(0,72)$ (YAKUBU \& MOHAMMED, 2012).

Tabela 2. Valores médios e Coeficientes de Variação das medidas corporais de 15 linhagens de ovinos da raça Santa Inês

\begin{tabular}{|c|c|c|c|c|c|c|}
\hline \multirow{2}{*}{ Linhagem } & \multicolumn{4}{|c|}{ Altura $(\mathrm{cm})$} & \multicolumn{2}{|c|}{ Circunferência (cm) } \\
\hline & Anterior & Tórax & Posterior & Pernas & Torácica & Canela \\
\hline L1 & $70,00^{\mathrm{e}}$ & $32,25^{\mathrm{d}}$ & $70,20^{\mathrm{e}}$ & $37,75^{\mathrm{b}}$ & $87,83^{\mathrm{d}}$ & $8,54^{\mathrm{b}}$ \\
\hline $\mathrm{L} 2$ & $72,17^{\mathrm{d}}$ & $33,90^{\mathrm{b}}$ & $72,12^{\mathrm{d}}$ & $38,26^{\mathrm{b}}$ & $92,24^{\mathrm{c}}$ & $8,54^{\mathrm{b}}$ \\
\hline L3 & $70,17^{\mathrm{e}}$ & $31,95^{\mathrm{d}}$ & $71,11^{\mathrm{d}}$ & $38,22^{\mathrm{b}}$ & $86,59^{\mathrm{d}}$ & $7,74^{\mathrm{d}}$ \\
\hline L4 & $73,29^{\mathrm{c}}$ & $33,27^{\mathrm{c}}$ & $73,48^{\mathrm{c}}$ & $40,01^{\mathrm{a}}$ & $90,98^{\mathrm{c}}$ & $8,39^{\mathrm{c}}$ \\
\hline L5 & $72,85^{\mathrm{c}}$ & $32,30^{\mathrm{d}}$ & $73,07^{\mathrm{c}}$ & $40,54^{\mathrm{a}}$ & $87,20^{\mathrm{d}}$ & $8,43^{\mathrm{c}}$ \\
\hline L6 & $70,84^{\mathrm{e}}$ & $33,02^{\mathrm{c}}$ & $71,52^{\mathrm{d}}$ & $37,82^{\mathrm{b}}$ & $89,84^{\mathrm{c}}$ & $8,30^{\mathrm{c}}$ \\
\hline L7 & $69,96^{\mathrm{e}}$ & $31,03^{\mathrm{e}}$ & $71,33^{\mathrm{d}}$ & $38,92^{\mathrm{b}}$ & $83,64^{\mathrm{e}}$ & $7,87^{\mathrm{d}}$ \\
\hline L8 & $74,98^{\mathrm{b}}$ & $33,87^{\mathrm{b}}$ & $75,17^{\mathrm{b}}$ & $41,10^{\mathrm{a}}$ & $91,10^{\mathrm{c}}$ & $8,31^{\mathrm{c}}$ \\
\hline L9 & $74,05^{\mathrm{c}}$ & $34,67^{b}$ & $74,28^{b}$ & $39,37^{\mathrm{a}}$ & $96,14^{\mathrm{b}}$ & $8,64^{\mathrm{b}}$ \\
\hline L10 & $69,78^{\mathrm{e}}$ & $31,75^{\mathrm{d}}$ & $71,42^{\mathrm{d}}$ & $38,00^{\mathrm{b}}$ & $85,58^{\mathrm{d}}$ & $7,85^{\mathrm{d}}$ \\
\hline L11 & $66,87^{\mathrm{f}}$ & $30,14^{\mathrm{f}}$ & $68,94^{\mathrm{e}}$ & $36,79^{\mathrm{c}}$ & $80,35^{\mathrm{f}}$ & $7,38^{\mathrm{e}}$ \\
\hline L12 & $71,65^{\mathrm{d}}$ & $33,48^{\mathrm{c}}$ & $71,90^{\mathrm{d}}$ & $38,12^{\mathrm{b}}$ & $91,54^{\mathrm{c}}$ & $8,46^{\mathrm{b}}$ \\
\hline L13 & $72,26^{\mathrm{d}}$ & $34,01^{\mathrm{b}}$ & $73,18^{\mathrm{c}}$ & $38,25^{\mathrm{b}}$ & $91,76^{\mathrm{c}}$ & $8,38^{\mathrm{c}}$ \\
\hline L14 & $68,43^{\mathrm{f}}$ & $32,22^{\mathrm{d}}$ & $69,12^{\mathrm{e}}$ & $36,20^{\mathrm{c}}$ & $87,48^{\mathrm{d}}$ & $8,27^{\mathrm{c}}$ \\
\hline L15 & $77,10^{\mathrm{a}}$ & $36,47^{\mathrm{a}}$ & $78,27^{\mathrm{a}}$ & $40,77^{\mathrm{a}}$ & $104,22^{\mathrm{a}}$ & $9,35^{\mathrm{a}}$ \\
\hline \multirow[t]{3}{*}{ CV (\%) } & 4,34 & 5,04 & 4,06 & 6,55 & 5,75 & 5,49 \\
\hline & \multicolumn{5}{|c|}{ Comprimento $(\mathrm{cm})$} & Largura \\
\hline & Corpo & Orelha & Face & Pescoço & Chanfro & $\begin{array}{c}\text { da face } \\
(\mathrm{cm})\end{array}$ \\
\hline L1 & $72,29^{d}$ & $18,07^{\mathrm{a}}$ & $22,00^{\mathrm{d}}$ & $34,11^{\mathrm{d}}$ & $17,30^{\mathrm{b}}$ & $13,78^{\mathrm{a}}$ \\
\hline L2 & $73,95^{\mathrm{c}}$ & $16,82^{\mathrm{c}}$ & $22,56^{\mathrm{c}}$ & $33,45^{\mathrm{d}}$ & $17,19^{\mathrm{b}}$ & $13,67^{\mathrm{b}}$ \\
\hline L3 & $73,91^{\mathrm{c}}$ & $16,33^{\mathrm{c}}$ & $21,77^{\mathrm{d}}$ & $36,04^{\mathrm{c}}$ & $16,83^{\mathrm{b}}$ & $13,26^{\mathrm{c}}$ \\
\hline L4 & $74,91^{\mathrm{b}}$ & $17,55^{\mathrm{a}}$ & $22,21^{\mathrm{c}}$ & $38,43^{\mathrm{a}}$ & $16,66^{\mathrm{c}}$ & $13,35^{\mathrm{c}}$ \\
\hline L5 & $73,58^{\mathrm{c}}$ & $17,67^{\mathrm{a}}$ & $21,42^{\mathrm{e}}$ & $38,16^{\mathrm{a}}$ & $16,04^{\mathrm{d}}$ & $13,00^{\mathrm{d}}$ \\
\hline L6 & $72,28^{\mathrm{d}}$ & $17,03^{\mathrm{b}}$ & $22,00^{\mathrm{d}}$ & $35,06^{\mathrm{c}}$ & $17,15^{\mathrm{b}}$ & $13,83^{\mathrm{a}}$ \\
\hline L7 & $72,83^{\mathrm{c}}$ & $17,00^{\mathrm{b}}$ & $21,48^{\mathrm{e}}$ & $33,61^{\mathrm{d}}$ & $16,46^{\mathrm{c}}$ & $13,19^{\mathrm{c}}$ \\
\hline L8 & $75,27^{\mathrm{b}}$ & $16,52^{\mathrm{c}}$ & $22,27^{\mathrm{c}}$ & $38,91^{\mathrm{a}}$ & $16,67^{\mathrm{c}}$ & $13,66^{\mathrm{b}}$ \\
\hline L9 & $77,07^{\mathrm{b}}$ & $17,20^{\mathrm{b}}$ & $23,08^{b}$ & $35,37^{\mathrm{c}}$ & $16,60^{\mathrm{c}}$ & $13,66^{\mathrm{b}}$ \\
\hline L10 & $72,03^{\mathrm{d}}$ & $16,73^{\mathrm{c}}$ & $21,42^{\mathrm{e}}$ & $35,16^{\mathrm{c}}$ & $16,98^{\mathrm{b}}$ & $13,45^{\mathrm{c}}$ \\
\hline L11 & $70,61^{\mathrm{d}}$ & $16,81^{\mathrm{c}}$ & $20,27^{\mathrm{f}}$ & $33,05^{\mathrm{d}}$ & $15,88^{\mathrm{d}}$ & $13,02^{\mathrm{d}}$ \\
\hline L12 & $73,54^{\mathrm{c}}$ & $17,51^{\mathrm{a}}$ & $22,71^{\mathrm{c}}$ & $35,34^{\mathrm{c}}$ & $17,66^{\mathrm{a}}$ & $13,83^{\mathrm{a}}$ \\
\hline L13 & $76,05^{\mathrm{b}}$ & $17,14^{\mathrm{b}}$ & $22,61^{\mathrm{c}}$ & $34,88^{\mathrm{c}}$ & $16,40^{\mathrm{c}}$ & $13,54^{\mathrm{b}}$ \\
\hline L14 & $71,93^{\mathrm{d}}$ & $17,16^{\mathrm{b}}$ & $22,00^{\mathrm{d}}$ & $34,12^{\mathrm{d}}$ & $17,20^{\mathrm{b}}$ & $13,70^{\mathrm{b}}$ \\
\hline L15 & $79,85^{\mathrm{a}}$ & $17,72^{\mathrm{a}}$ & $23,58^{\mathrm{a}}$ & $36,56^{\mathrm{b}}$ & $17,31^{\mathrm{b}}$ & $13,99^{\mathrm{a}}$ \\
\hline CV (\%) & 4,72 & 6,02 & 4,26 & 6,85 & 4,93 & 3,25 \\
\hline
\end{tabular}

*Médias seguidas de letras diferentes na coluna diferem entre si ao nível de 5,0\% de probabilidade pelo teste Scott-knott. 
Rev. Bras. Saúde Prod. Anim., Salvador, v.16, n.4, p.784-795 out../dez.., 2015 http://www.rbspa.ufba.br ISSN 15199940

Tabela 3. Correlações entre medidas corporais de ovinos da raça Santa Inês

\begin{tabular}{llllllllllll}
\hline & $\mathrm{AT}$ & $\mathrm{AP}$ & $\mathrm{CC}$ & $\mathrm{CT}$ & $\mathrm{CN}$ & $\mathrm{CP}$ & $\mathrm{CH}$ & $\mathrm{CO}$ & $\mathrm{LF}$ & $\mathrm{CF}$ & PR \\
\hline $\mathrm{AA}$ & $0,69^{*}$ & $0,91^{*}$ & $0,69^{*}$ & $0,64^{*}$ & $0,51^{*}$ & $0,50^{*}$ & $0,20^{*}$ & $0,22^{*}$ & $0,25^{*}$ & $0,57^{*}$ & $0,84^{*}$ \\
$\mathrm{AT}$ & - & $0,65^{*}$ & $0,66^{*}$ & $0,85^{*}$ & $0,61^{*}$ & $0,31^{*}$ & $0,32^{*}$ & $0,16^{*}$ & $0,48^{*}$ & $0,68^{*}$ & $0,18^{*}$ \\
$\mathrm{AP}$ & - & - & $0,71^{*}$ & $0,64^{*}$ & $0,52^{*}$ & $0,50^{*}$ & $0,21^{*}$ & $0,23^{*}$ & $0,25^{*}$ & $0,56^{*}$ & $0,75^{*}$ \\
$\mathrm{CC}$ & - & - & - & $0,63^{*}$ & $0,49^{*}$ & $0,33^{*}$ & $0,18^{*}$ & $0,22^{*}$ & $0,28^{*}$ & $0,58^{*}$ & $0,44^{*}$ \\
$\mathrm{CT}$ & - & - & - & - & $0,70^{*}$ & $0,28^{*}$ & $0,34^{*}$ & $0,17^{*}$ & $0,55^{*}$ & $0,67^{*}$ & $0,22^{*}$ \\
$\mathrm{CN}$ & - & - & - & - & - & $0,25^{*}$ & $0,39^{*}$ & $0,35^{*}$ & $0,53^{*}$ & $0,63^{*}$ & $0,23^{*}$ \\
$\mathrm{CP}$ & - & - & - & - & - & - & $0,10^{\mathrm{ns}}$ & $0,14^{*}$ & $0,08^{\mathrm{ns}}$ & $0,24^{*}$ & $0,44^{*}$ \\
$\mathrm{CH}$ & - & - & - & - & - & - & - & $0,29^{*}$ & $0,54^{*}$ & $0,61^{*}$ & $0,02^{\mathrm{ns}}$ \\
$\mathrm{CO}$ & - & - & - & - & - & - & - & - & $0,18^{*}$ & $0,33^{*}$ & $0,18^{*}$ \\
$\mathrm{LF}$ & - & - & - & - & - & - & - & - & - & $0,58^{*}$ & $0,02^{\mathrm{ns}}$ \\
$\mathrm{CF}$ & - & - & - & - & - & - & - & - & - & - & $0,26^{*}$ \\
\hline
\end{tabular}

$\mathrm{AA}=$ Altura Anterior; $\mathrm{AP}=$ Altura Posterior; $\mathrm{AT}=$ Altura do Tórax $\mathrm{CC}=$ Comprimento do Corpo; $\mathrm{CT}$ = Circunferência Torácica; $\mathrm{CN}=$ Circunferência de Canela; $\mathrm{CP}=$ Comprimento de Pescoço; $\mathrm{CH}=$ Chanfro; $\mathrm{CO}=$ Comprimento de Orelha; $\mathrm{LF}=$ Largura da Face; $\mathrm{CF}=$ Comprimento da Face; $\mathrm{PR}=$ Altura das pernas.

*Valores significativos $(\mathrm{p}<0,01)$.

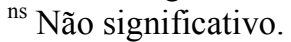

No processo de melhoramento de uma raça, é imprescindível que todas as medidas biométricas e as suas possíveis inter-relações sejam consideradas na definição dos critérios de seleção (PESMEN \& YARDIMCI, 2008). Nesse sentido, De La Barra et al. (2014) salientam que a seleção baseada na conformação tem sido amplamente utilizada como ferramenta para melhorar a produtividade na ovinocultura. No presente estudo, as correlações positivas e significativas entre $\mathrm{CN}$ e AA, AT, AP, CC e CT sugerem que a utilização da maior $\mathrm{CN}$ como critério de seleção pode proporcionar a obtenção de animais mais produtivos.

$\mathrm{Na}$ primeira análise discriminante, as seis últimas Variáveis Canônicas (VC) apresentaram autovalor inferior a 0,7, sugerindo que até seis medidas poderiam ser descartadas. No entanto, foram descartadas apenas duas medidas (Altura Anterior e Altura das Pernas), uma vez que a Altura Anterior apresentou o maior autovetor em cinco
VC, enquanto que a Altura das Pernas apenas em uma.

Das três medidas relacionadas à estatura animal, apenas a Altura Posterior foi mantida, indicando que, em futuros estudos, apenas essa medida é suficiente para caracterização da estatura. O descarte das medidas Altura Anterior e Altura das Pernas não provocou perda de informação, devido às elevadas correlações $(r>0,7)$ com algumas das medidas mantidas (CC, CT e AP).

Após o descarte, as três primeiras $\mathrm{VC}$ representaram, de forma conjunta, $81 \%$ da diversidade fenotípica entre linhagens. $\mathrm{O}$ descarte das medidas Altura Anterior e Altura das Pernas levou ao acréscimo de $2,97 \%$ em relação às três $\mathrm{VC}$ obtidas anteriormente. Individualmente, as três primeiras VC representaram 36,51\%, $32,02 \%$ e $12,47 \%$ da variância total, respectivamente.

A importância da VC depende das heterogeneidades inter (variâncias e covariâncias fenotípica) e intrapopulacional (variâncias e covariâncias residuais). Quanto menor a 
Rev. Bras. Saúde Prod. Anim., Salvador, v.16, n.4, p.784-795 out../dez.., 2015 http://www.rbspa.ufba.br

heterogeneidade inter-pupulacional e maior intra-populacional, maior será o número de $\mathrm{VC}$ necessárias para explicar a diversidade total. Em estudo com os ovinos locais em Burkina Faso, 100\% da variância total foi explicada pelas duas primeiras VC (TRAORÉ et al., 2008), enquanto que em ovinos espanhóis, as três primeiras VC explicaram apenas $60,20 \%$ da variância total (LEGAZ et al., 2011).
Em relação à variabilidade intrapopulacional, a linhagem 15 , apesar de concentrar todos os seus indivíduos em um único quadrante, apresentou maior dispersão que as linhagens 1, 10 e 11, as quais mostraram maior homogeneidade. As linhagens 3, 6, 9 e 12 também apresentaram grande variabilidade, sendo que a linhagem 6 foi a mais dispersa, uma vez que apresentou número semelhante de indivíduos em todos os quadrantes (Figura 1).

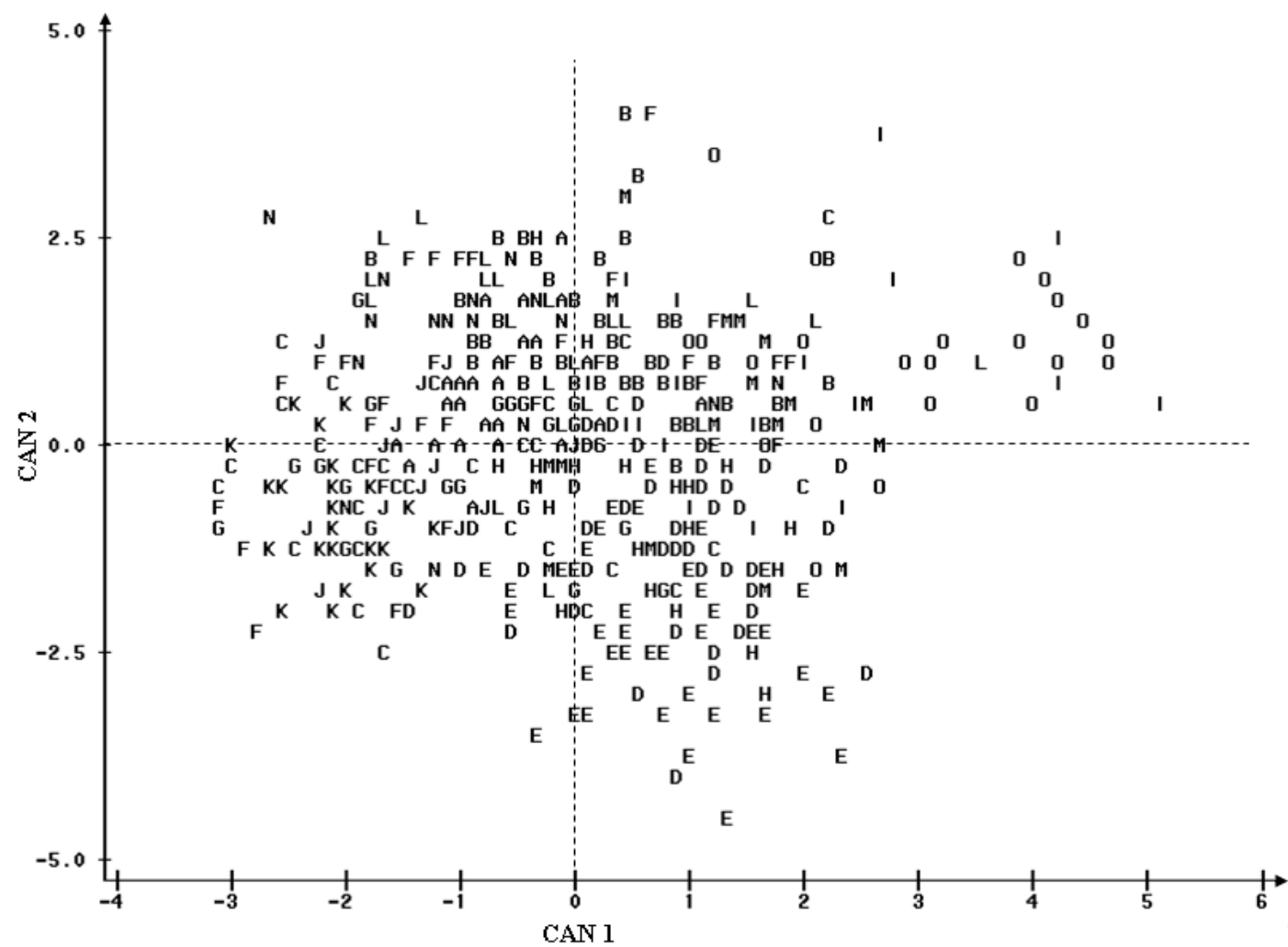

$\mathrm{A}=$ Linhagem 1; $\mathrm{B}=$ Linhagem 2; $\mathrm{C}=$ Linhagem 3; $\mathrm{D}=$ Linhagem 4; $\mathrm{E}=$ Linhagem 5; $\mathrm{F}=$ Linhagem 6; $\mathrm{G}=$ Linhagem 7; $\mathrm{H}=$ Linhagem 8; $\mathrm{I}=$ Linhagem 9; $\mathrm{J}=$ Linhagem 10; $\mathrm{K}=$ Linhagem 11; $\mathrm{L}=$ Linhagem 12; $\mathrm{M}=$ Linhagem 13; $\mathrm{N}=$ Linhagem 14; $\mathrm{O}=$ Linhagem 15.

Figura 1. Dispersão gráfica dos indivíduos das 15 linhagens de ovinos da raça Santa Inês em relação às Variáveis Canônicas 1 e 2

Dentre todas as linhagens, as maiores distâncias ocorreram entre a 5, 11 e 15 , enquanto que as linhagens 6 e 12 apresentaram as menores médias de distâncias em relação às demais linhagens
(Tabela 4). Essa heterogeneidade das distâncias entre as linhagens não deve ser compreendida como reflexo exclusivo da diversidade de genótipos que deram origem à raça Santa Inês, mas também, 
como consequência de fatores ambientais e de seleção a que os animais foram submetidos por diferentes criadores ao longo das gerações.

Considerando que genitores de bom desempenho, com certo grau de diversidade, possam apresentar constituição genética complementar que proporciona maior produtividade a $\mathrm{F}_{1}$ (YAKUBU et al., 2011), o acasalamento de indivíduos entre as linhagens 5, $11 \mathrm{e}$ 15 pode proporcionar a obtenção de animais com maior produtividade, em virtude do incremento causado pelo fenômeno da heterose, principalmente em relação às características relacionadas à produção de carne, Circunferência Torácica, Comprimento do Corpo e Altura do Tórax. Evidentemente que esse procedimento demanda definição prévia de critérios norteadores do processo de seleção. Bem como, do mérito genético de cada genitor em relação às características de importância econômica.

Tabela 4. Matriz das distâncias generalizadas de Mahalanobis $\left(\mathrm{D}^{2}\right)$ entre as linhagens de ovinos da raça Santa Inês

\begin{tabular}{ccccccccccccccc}
\hline & L2 & L3 & L4 & L5 & L6 & L7 & L8 & L9 & L10 & L11 & L12 & L13 & L14 & L15 \\
\hline L1 & 4,49 & 9,04 & 7,68 & 10,60 & 2,40 & 5,63 & 11,01 & 9,69 & 5,14 & 8,67 & 2,27 & 7,15 & 1,44 & 15,46 \\
L2 & - & 7,14 & 7,86 & 13,23 & 2,53 & 6,01 & 8,19 & 3,60 & 5,95 & 12,00 & 1,83 & 2,88 & 2,94 & 8,46 \\
L3 & - & - & 5,41 & 10,59 & 4,92 & 3,03 & 5,25 & 8,91 & 2,11 & 5,27 & 5,29 & 6,15 & 4,92 & 19,55 \\
L4 & - & - & - & 2,13 & 6,11 & 6,44 & 3,24 & 5,24 & 6,58 & 10,77 & 5,63 & 4,28 & 7,26 & 11,03 \\
L5 & - & - & - & - & 11,03 & 8,64 & 6,98 & 10,55 & 10,50 & 11,93 & 11,61 & 8,52 & 12,09 & 16,39 \\
L6 & - & - & - & - & - & 4,43 & 5,26 & 7,03 & 2,00 & 7,22 & 1,05 & 4,80 & 1,34 & 13,13 \\
L7 & - & - & - & - & - & - & 7,31 & 8,67 & 1,98 & 2,41 & 5,55 & 5,05 & 4,49 & 18,68 \\
L8 & - & - & - & - & - & - & - & 6,90 & 5,36 & 11,68 & 6,81 & 5,12 & 8,96 & 12,88 \\
L9 & - & - & - & - & - & - & - & - & 10,85 & 16,32 & 5,58 & 1,02 & 8,17 & 4,45 \\
L10 & - & - & - & - & - & - & - & - & - & 3,08 & 3,75 & 7,18 & 3,28 & 19,47 \\
L11 & - & - & - & - & - & - & - & - & - & - & 10,41 & 10,76 & 7,31 & 28,88 \\
L12 & - & - & - & - & - & - & - & - & - & - & - & 4,38 & 1,24 & 11,03 \\
L13 & - & - & - & - & - & - & - & - & - & - & - & - & 5,63 & 8,16 \\
L14 & - & - & - & - & - & - & - & - & - & - & - & - & - & 16,57 \\
\hline
\end{tabular}

O ponto de corte do dendrograma, definido pela média da matriz de dissimilaridade $(7,50)$, possibilitou a formação de três grupos (Figura 2). $\mathrm{O}$ primeiro grupo contendo 11 linhagens $(1 ; 2 ; 3 ; 6 ; 7 ; 9,10 ; 11 ; 12 ; 13$ e 14$)$, com indivíduos de menor porte. $\mathrm{O}$ segundo grupo constituído por três linhagens $(4,5$ e 8$)$ cujos indivíduos apresentaram as medidas corporais medianas. O terceiro grupo, composto apenas pela linhagem 15 caracterizada por apresentar indivíduos com maiores dimensões.
Os animais do primeiro grupo, apesar das dimensões medianas para a maioria das medidas corporais, apresentaram altos valores para Comprimento de Perna. Considerando que animais altos tendem a ser tardios (COSTA JÚNIOR et al., 2006), os animais integrantes dessas linhagens devem ser incluídos em futuros programas de seleção da raça, uma vez que essa característica pode se constituir em limitação para alcance de maior produtividade de carne. 
Rev. Bras. Saúde Prod. Anim., Salvador, v.16, n.4, p.784-795 out../dez.., 2015 http://www.rbspa.ufba.br ISSN 15199940

Por outro lado, enquanto o maior tamanho corporal do terceiro grupo suscita maiores necessidades nutricionais, o menor porte do primeiro grupo pode ser indicativo de maior precocidade no crescimento (MALHADO et al., 2008). Dessa forma, o acasalamento criterioso entre as diferentes linhagens pode ser proposto como diretriz para compatibilizar produtividade e adaptabilidade em função das condições ambientais e do sistema de produção.
No que ser refere à acurácia do agrupamento das linhagens, o coeficiente de correlação cofenética foi de 74,38\%. Considerando que o coeficiente de correlação cofenética equivale à correlação de Pearson entre a matriz de dissimilaridade original e aquela obtida após a construção do dendrograma, quanto mais próximo de um, menor será a distorção provocada pelo agrupamento das linhagens.

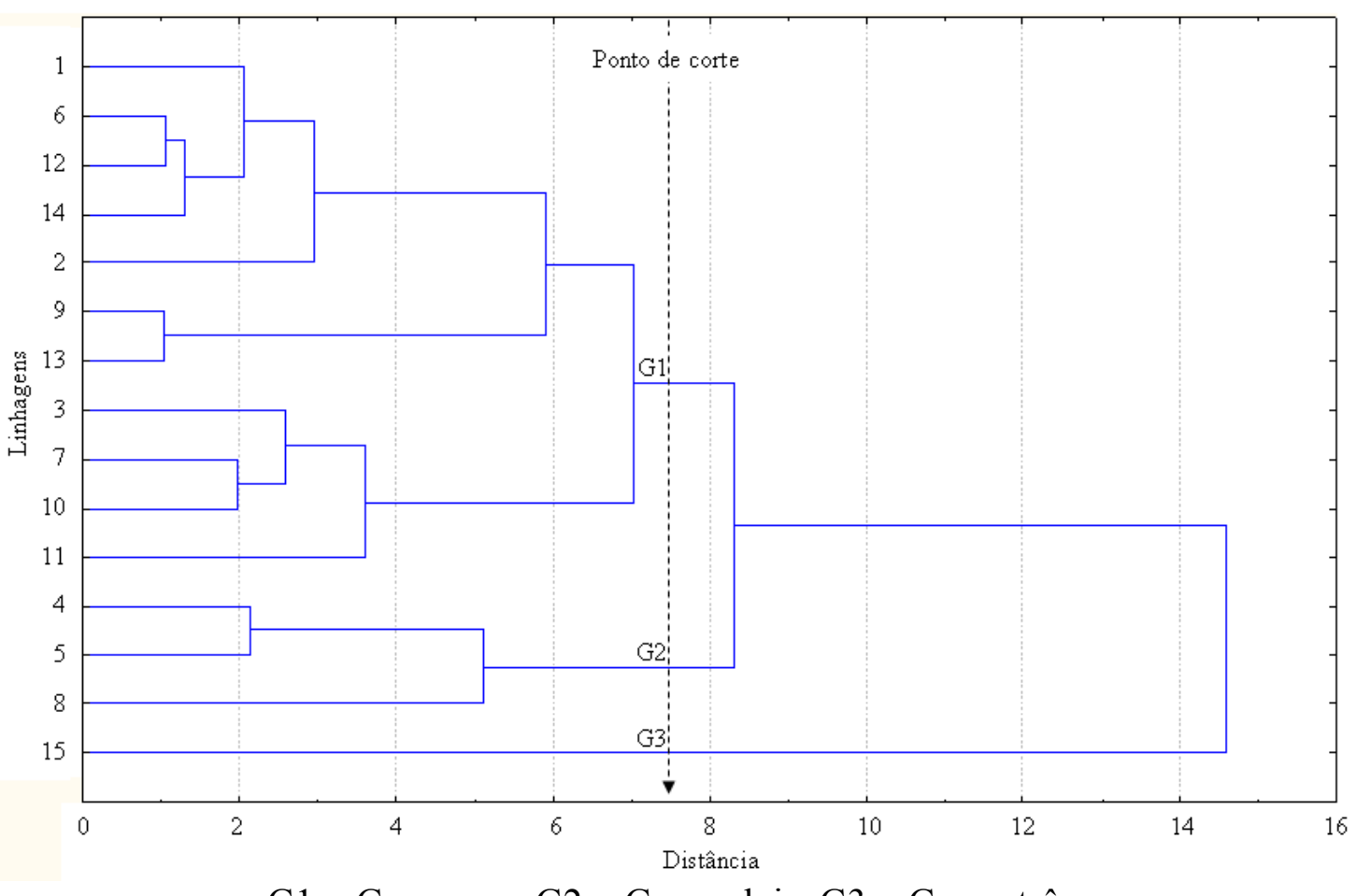

G1 = Grupo um; G2 = Grupo dois; G3 = Grupo três .

Figura 2. Dendrograma das linhagens de ovinos da raça Santa Inês, obtido pelo método UPGMA

Todas as medidas corporais contribuíram, em maior ou menor proporção, para a determinação da divergência fenotípica entre as linhagens. Enquanto que as medidas ligadas à produção contribuíram com $53,40 \%$ da diversidade entre as linhagens, aquelas relacionadas ao padrão racial contribuíram com 46,59\%. As cinco medidas de maior importância contribuíram com $76,89 \%$ da diversidade entre as linhagens, das quais, três relacionadas ao padrão racial e duas à produção (Tabela 5). 
Rev. Bras. Saúde Prod. Anim., Salvador, v.16, n.4, p.784-795 out../dez.., 2015 http://www.rbspa.ufba.br ISSN 15199940

Tabela 5. Importância relativa das medidas corporais $\left(\mathrm{S}_{\mathrm{j}}\right)$ para diversidade fenotípica em linhagens de ovinos da raça Santa Inês

\begin{tabular}{lc}
\hline Medidas corporais & S.j (\%) \\
\hline Circunferência torácica & 20,53 \\
Circunferência de canela & 19,49 \\
Comprimento de face & 14,87 \\
Comprimento de pescoço & 14,37 \\
Comprimento de chanfro & 7,63 \\
Altura posterior & 7,08 \\
Altura do tórax & 5,69 \\
Comprimento de orelha & 5,24 \\
Largura de face & 4,48 \\
Comprimento do corpo & 0,61 \\
\hline
\end{tabular}

O padrão racial é utilizado como importante parâmetro na avaliação e inclusão dos animais controlados nos livros de registro, mas a seleção baseada apenas nas características relacionadas ao aspecto racial não promovem elevação da produção de carne. Nesse sentido, Cezar \& Sousa (2010) salientam que a utilização das medidas ligadas à produção como critério de seleção promove $o$ incremento no rendimento de carcaça.

Assim, as linhagens de ovinos Santa Inês apresentam elevada diversidade fenotípica, sendo que as características relacionadas à produção e a caracterização racial apresentam importância semelhante na diferenciação entre linhagens. A diversidade devida às medidas de caracterização racial sugere que as linhagens foram submetidas a pressões seletivas distintas, remetendo à necessidade de definição de objetivos e critérios de seleção para os indivíduos da raça Santa Inês.

\section{REFERÊNCIAS}

BIAGIOTTI, D.; SARMENTO, J.L.R.; Ó, A.O.; RÊGO NETO, A.A.;

SANTOS, G. V.; SANTOS, N.P.S.; TORRES, T.S.; NERI, V.S.

Caracterização fenotípica de ovinos da raça Santa Inês no Estado do Piauí.

\section{Revista Brasileira de Saúde e}

Produção Animal [online], v.14, n.1, p.29-42, 2013.

BIAGIOTTI, D.; GUIMARÃES, F.F.; SARMENTO, J.L.R.; SANTOS, G. V.; RÊGO NETO, A.A.; SANTOS, N.P.S.; SARAIVA, T.T.; FIGUEIREDO FILHO, L.A.S.; SENA, L.S. Uso de estatística multivariada para estudo de caracterização racial de ovinos. Acta Tecnológica, v.9, n.1, p.16-26, 2014.

CARNEIRO, P.L.S.; MALHADO, C.H.M.; AZEVEDO, D.M.M.R.; SOUZA, L.G.R.; SILVA, D.C.; ALVES, A.A.; CAMPELO, J.E.G.;COSTA JÚNIOR, G.S. Estudo de populações de Ovinos Santa Inês utilizando técnicas de análise multivariada. Revista Científica de Produção Animal, v.8, n.1, 2006. 
Rev. Bras. Saúde Prod. Anim., Salvador, v.16, n.4, p.784-795 out../dez.., 2015 http://www.rbspa.ufba.br ISSN 15199940

CEZAR, M.F.; SOUSA, W.H. Proposta de avaliação e classificação de carcaças de ovinos deslanados e caprinos.

Tecnologia \& Ciência Agropecuária, v.4, n.4, p.41-51, 2010.

COSTA JÚNIOR, G.S.; CAMPELO, J.E.G.; AZEVÊDO, D.M.M.R.; MARTINS FILHO, R.; CAVALCANTE, R.R.; LOPES, J.B.; OLIVEIRA, M.E.D. Caracterização morfométrica de ovinos da raça Santa Inês criados nas microrregiões de Teresina e Campo Maior, Piauí. Revista Brasileira de Zootecnia, v.35, n.6, p.2260-2267, 2006.

\section{CRUZ, C.D. Programa Genes:} aplicativo computacional em genética. Versão 6.0. Viçosa, MG: Universidade Federal de Viçosa, 2008.

DARAMOLA, J.O.; ADELOVE, A.A. Physiological adaptation to the humid tropics with special reference to the West African Dwarf (WAD) goat, Tropical Animal Health and Production, v.41, p.1005-1016, 2009.

DE LA BARRA, R.; LATORRE, E.; MARTÍNEZ, M.E.; CALDERÓN, C. Morphostructural Differentiation and Variability of Merino Sheep Breed Under Sustained Directional Selection.

International Journal of Morphology, v.32, n.3, p.1069-1073, 2014.

DOSSA, L.H.; WOLLNY, C.; GAULY, $M$. Spatial variation in goat populations from Benin as revealed by multivariate analysis of morphological traits. Small Ruminant Research, v.73, p.150-159, 2007.

GILBERT, R.P.; BAILEY, D.R.; SHANNON, N.H. Body dimensions and carcass measurements of cattle selected for postweaning gain fed two different diets. Journal Animal Science, v.71, p.1688-1698, 1993.
JOLLIFFE, I. T. Discarding variables in a principal component analysis. II. Real data. Applied Statistics, v.22, p.21-31, 1972.

LEGAZ, E.; CERVANTES, I.; PÉREZCABAL, M.A.; DE LA FUENTE, L.F.; MARTINEZ, R., GOYACHE, F.; GUTIÉRREZ, J.P. Multivariate characterisation of morphological traits in Assaf (Assaf.E) Sheep. Small Ruminant Research, v.100, p.122130, 2011.

MALHADO, C.H.M.; CARNEIRO, P.L.S.; CRUZ, J.F.; OLIVEIRA, D.F.D.; AZEVEDO, D.M.M.R.; ROCHA, J.L. Curvas de crescimento para caprinos da raça Anglo-Nubiana criados na caatinga: rebanho de elite e comercial. Revista Brasileira de Saúde e Produção Animal, v.9, n.4, p.662671, 2008.

PAIVA, S.R.; SILVÉRIO, V.C.; EGITO, A.A.; MCMANUS, C.; FARIA, D.A.; MARIANTE, A.S.; CASTRO, S.R.; ALBUQUERQUE, M.S.M.; DERGAM, J.A. Genetic variability of the Brazilian hair sheep breeds. Revista Agropecuária Brasileira, v.40, n.9, p.887-893, 2005.

PESMEN, G.; YARDIMCI, M.

Estimating the live weight using some body measurements in Saanen goats. Archiva Zootechnica, v.11, n.4, p.3040, 2008.

PIRES, L.C.; MACHADO, T.M.M.; ARAÚJO, A.M.; SILVA, J.B.L.; EUCLYDES, R.F.; COSTA, M.S.; OLSON, T.A. Cluster evaluation of Brazilian and Moroccan goat populations using physical measurements. Revista Brasileira de Zootecnia, v.42, n.10, p.713-720, 2013. 
Rev. Bras. Saúde Prod. Anim., Salvador, v.16, n.4, p.784-795 out../dez.., 2015 http://www.rbspa.ufba.br

SAS/STAT. User's guide. Versão 8.0.

Cary: SAS Institut Inc., 2003.

SEARLE, T.W.; GRAHAM, M.C.; DONNELLY, J.B. Change of skeletal dimensions during growth in sheep: the effect of nutrition. Journal of Agricultural Science, v.112, n.3, p.321-327, 1989.

SINGH, D. The relative importance of characters affecting genetic divergence. The Indian Journal of Genetic and Plant Breeding, v.41, n.1, p.237-245, 1981.

TORRES FILHO, R.A.; EUCLYDES, R.F.; TORRES, R.A.; LOPES, P.S.; BREDA, F.C. Estudo da divergência genética entre linhas de suínos utilizando técnicas de análise multivariada. Arquivo Brasileiro de Medicina Veterinária e Zootecnia, v.57, n.3, p.390-395, 2005.

TRAORÉ, A.; TAMBOURA, H.H.; KABORÉ, A.; ROYO, L.J.; FERNÁNDEZ, I.; ÁLVAREZ, I.; SANGARÉ, M.; BOUCHEL, D.; POIVEY, J.P.; FRANCOIS, D.; TOGUYENI, A.; SAWADOGO, L.; GOYACHE, F. Multivariate characterization of morphological traits in Burkina Faso sheep. Small ruminant research, v.80, p.62-67, 2008.

YAKUBU, A.; SALAKO, A.E.; IMUMORIN, I.G. Comparative multivariate analysis of biometric traits of West African Dwarf and Red Sokoto goats. Tropical Animal Health and

Production, v.43, n.3, p.561-566, 2011.

YAKUBU, A.; IBRAHIM, I.A.

Multivariate analysis of morphostructural characteristics in Nigerian indigenous sheep. Italian Journal of Animal Science, v.10, n.17, 2011.
YAKUBU, A.; MOHAMMED, G. L. Application of path analysis methodology in assessing the relationship netween body weight and biometric traits of Red Sokoto goats in Northern Nigeria. Biotechnology in Animal Husbandry, v.28, n.1, p.107117, 2012.

Data de recebimento: 05/07/2015

Data de provação: 14/10/2015 